\title{
IDENTIFICAÇÃO DOS INSETOS INFESTANTES DE ALIMENTOS ATRAVÉS DA MICROMORFOLOGIA DE SEUS FRAGMENTOS ${ }^{1}$
}

\author{
Carlos Henrry Bellot Vargas ${ }^{2}$ \\ Armando Antunes de Almeida ${ }^{2}$
}

\begin{abstract}
IDENTIFICATION OF INSECT CONTAMINANTS OF FOOD BY THE MICROMORPHOLOGY OF THE INSECT FRAGMENTS. The identification of insect fragments showed that the infestation was done, mainly, by Coleoptera of the genus Sitophilus Schönherr, 1838 (Curculionidae) and Rhizopertha dominica (Fabricius, 1792) (Bostrychidae), that are caracteristic of internal infestation, and in lesser percentage by insects characteristic of external infestation, as Crytolestes spp. (Cucujidae), Tribolium spp. (Tenebrionidae), Oryzaephilus surinamensis (Linnaeus, 1758) (Cucujidae) and Lasioderma serricorne (Fabricius, 1792) (Anobiidae). From the total of recovered and identified insect fragments, $59 \%$ were fragments of elytra, $23 \%$ of mandibles, and in minor percentage by tarsal segments, claws, tibial spurs, legs, tarsus, eyes, antennae, exuviae and larvae urogomphi.

KEY WORDS. Insect fragments, food, identification, micromorphology
\end{abstract}

Os produtos agrícolas armazenados, principalmente, o trigo e seus sub-produtos, como as farinhas, massas alimentícias (macarrão) e outros que têm como principal matéria prima a farinha de trigo, podem apresentar contaminação biológica devido à infestação dos grãos de trigo por insetos pragas do campo ou dos armazéns.

Particularmente, no caso de contaminação por matérias estranhas nos derivados de grãos de trigo, as indústrias moageiras estão equipadas para minimizar esta contaminação, pois, antes da moagem os grãos passam por um rigoroso processo de limpeza. No entanto, quando os grãos apresentam infestação interna por insetos adultos e/ou suas formas imaturas, e não sendo possível eliminar este tipo de infestação os mesmos serão moídos junto com os grãos, conseqüentemente, a farinha proveniente destes grãos estará contaminada com fragmentos de insetos.

A evidência da contaminação dos alimentos industrializados por insetos é devida, normalmente, à presença de pequenos fragmentos do exoesqueleto, mas podem aparecer insetos adultos e larvas inteiras; 'são também encontrados outros contaminantes, como ácaros e pêlos de roedores.

Durante a moagem, o transporte, a mistura ou o processamento da matéria prima, os insetos são, geralmente, quebrados em pequenos fragmentos. Assim a identificação e a dedução da origem do material estranho é feita através destes fragmentos, como pedaços de élitros, do tórax, de mandíbulas, de pernas, de antenas, de cápsulas cefálicas e só raramente dos insetos inteiros.

1) Contribuição número 902 do Departamento de Zoologia, Universidade Federal do Paraná.

2) Departamento de Zoologia, Universidade Federal do Paraná. Caixa Postal 19020, 81531-990 Curitiba, Paraná, Brasil. Bolsistga do CNPq. 



Fig. 1. Sitophilus spp. adulto. (a) Cabeça, (b) antena, (c) antenômero apical, (d) antenômeros, (e) fragmentos dos olhos compostos, (f) mandibulas (esquerda e direita).


Fig. 2. Sitophilus spp. adulto. (a) Perna completa; Fragmentos: (b) da coxa, (c) do fêmur, (d) do trocânter, (e) da tíbia, (f) do tarso. 
O primeiro trabalho publicado, que abrange a identificação dos fragmentos de insetos como tais e a determinação dos insetos dos quais eles são derivados, foi realizado por HARRIS (1950). Segundo este autor, os caracteres de identificação da cutícula se baseiam na ausência de estruturas celulares, na translucidez, nas cerdas, nas bases das cerdas, nos espinhos e nos padrões da superfície do tegumento. Cita, também, as características peculiares dos fragmentos de insetos que diferenciam das partículas alimentares; assim como, os diferentes grupos de insetos que podem ocorrer nos alimentos através da infestação no campo, mista (campo e armazém), exclusiva de armazéns, e da infestação de outros insetos contaminantes acidentais.

HEUERMANN \& KURTZ (1955) observaram o élitro e consideraram as formações que constituem a sua superfície como padrões para a sua identificação, pois apresentam formas estruturais específicas.

Segundo JACKSON et al. (1956), os artículos antenais isolados ou fragmentos destes, mais característicos e que geralmente são mais rapidamente reconhecidos, estão localizados na região flagelar.

KURTZ (1956) fornece uma lista dos fragmentos comumente encontrados como contaminantes, correspondentes a 12 espécies de larvas de coleópteros. As regiões mais estudadas e fotomicrografadas correspondem à cápsula cefálica e ao apêndice caudal.

THRASHER \& KURTZ (1957) observaram que as pernas dos adultos raramente são recuperadas intactas dos alimentos; geralmente, são separadas em um, dois ou mais artículos ou como fragmentos de um artículo; neste último caso, podem ser identificados, apenas, se o fragmento recuperado apresentar características que são diagnósticos de um segmento intacto.

Segundo JACKSON et al. (1958) e MCCLYMONT PEACE (1985) as mandíbulas, em razão de sua rigidez, resistem à trituração podendo ser extraídas dos alimentos numa condição relativamente intacta, e são encontradas em maiores proporções entre os fragmentos totais, sendo facilmente identificadas ao microscópio estereoscópico. Estes autores elaboraram chaves, ilustradas com fotomicrografias e desenhos, para a identificação de mandíbulas de insetos adultos e de larvas comumente encontradas em produtos alimentícios armazenados.

GoRHAM $(1975,1979)$ conseguiu sintetizar os trabalhos realizados por dezenas de pesquisadores sobre a importância para a saúde humana da presença de insetos e/ou seus fragmentos nos gêneros alimentícios. Segundo este autor, comparando a quantidade de informações disponíveis sobre doenças originadas por alimentos em geral, parece ser relativamente pequeno o conhecimento acerca de enfermidades associadas a este tipo de contaminação.

Segundo WoODBURY (1983) os fragmentos identificáveis contribuem para a identificação da espécie da qual se originaram, sendo a chave para o diagnóstico da etiologia da contaminação, fato este, que valoriza a análise, uma vez que se pode chegar à conclusão em que etapa da produção o alimento foi contaminado.

O objetivo deste trabalho é o de determinar as espécies de insetos infestantes, através da identificação dos fragmentos recuperados nos produtos alimentícios analisados (farinhas de trigo especial, comum e macarrão), além de determinar a 



Fig. 3. Larva de Sitophilus spp. (a) Fragmentos da gena; (b) da fronte, (c-d) mandíbulas.
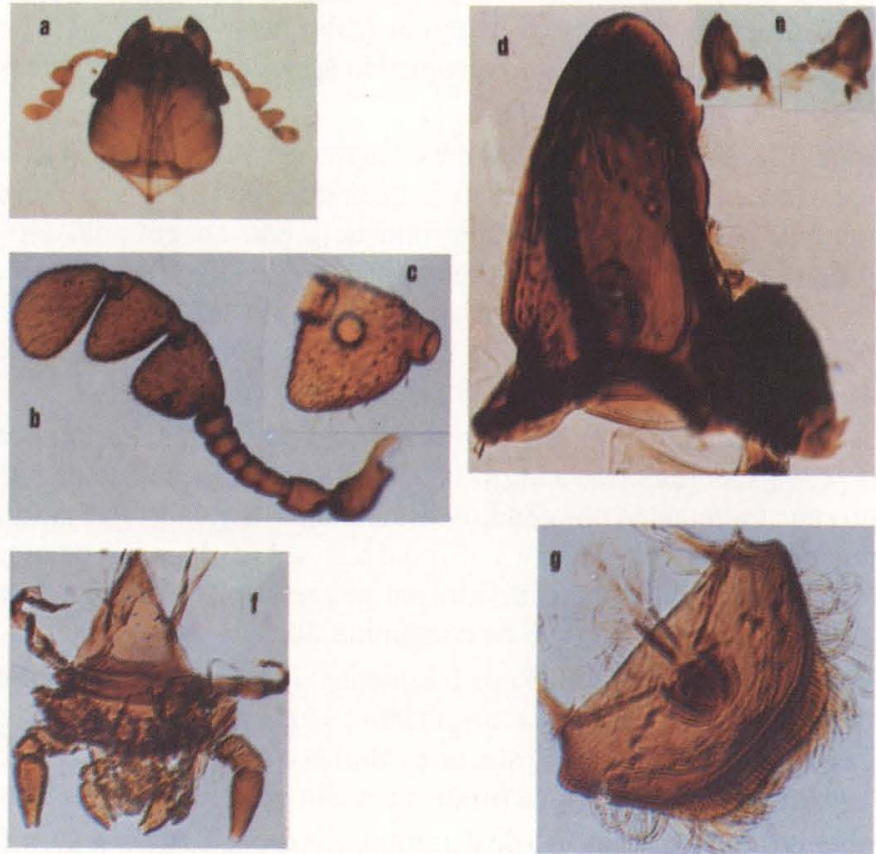

Fig.4. Rhizopertha dominica (Fabricius, 1792) adulto. (a) Cabeça, (b) antena, (c) antenômero, (d) mandíbulas esquerda, (e) mandibulas esquerda e direita, (f) lábio, (g) labro. 
porcentagem de ocorrência dos diferentes tipos de fragmentos e de seu tamanho. Assim como a obtenção de fotografias para a elaboração de um guia ilustrativo de apoio para os analistas de alimentos.

\section{MATERIAL E MÉTODOS}

A extração dos fragmentos foi feita através do método da hidrólise ácida (técnicas $n^{\circ} 972.32$ e 969.41) descritas na AOAC (1990). O reconhecimento e a identificação dos fragmentos de insetos encontrados nas massas alimentícias (macarrão) e nas farinhas de trigo comum e especial produzidas e comercializadas em Curitiba, Paraná, foi feita através da comparação com as regiões do corpo de espécimens previamente identificados e montados em lâminas permantentes. As lâminas foram montadas segundo a metodologia descrita por GoRHAM (1977).

A quantificação da freqüência de ocorrência dos fragmentos das diferentes partes do corpo do inseto, assim como a sua mensuração determinando-se o tamanho máximo, médio e mínimo, foram realizadas. Para isto mensurou-se 1146 fragmentos com auxílio de um microscópio estereoscópico com ocular micrométrica.

As lâminas permanentes das principais espécies e dos fragmentos encontrados foram fotografados nos Departamentos de Zoologia e de Genética da Universidade Federal do Paraná, utilizando-se uma câmara fotográfica acoplada a microscópios estereoscópico e óptico.

\section{RESULTADOS E DISCUSSÃO}

Os fragmentos de insetos identificados foram originários de espécies de coleópteros pragas, características de infestação interna do grão, correspondentes ao gênero Sitophilus spp. (Curculionidae) (Figs 1-3), e à espécie Rhizopertha dominica (Fabricius, 1792) (Bostrychidae) (Figs 4-5), tendo sido encontrados em $100 \%$ e $74,07 \%$, respectivamente, das amostras analisadas, e pelas espécies características de contaminação externa, geralmente, espécies que contaminam os produtos nos armazéns, nos silos, nos moinhos e/ou nos locais de processamento, como Cryptolestes spp. (Cucujidae) (Figs 6-7), Tribolium spp. (Tenebrionidae), Oryzaephilus surinamensis (Linnaeus, 1758) (Cucujidae), Lasioderma serricorne (Fabricius, 1792) (Anobiidae) e os psocópteros Liposcelis spp., cujos fragmentos foram encontrados em $55,55 \%, 25,92 \%, 7,41 \%, 3,70 \%$ e $6,1 \%$, respectivamente, das amostras analisadas.

O fato de serem os fragmentos encontrados, mais frequentemente, originários de Sitophilus spp. e de Rhyzopertha dominica, foi, também, constatado por GECAN \& ATKINSON (1985), LOCATELLI \& CODOVILli (1986) e BONAFACCIA et al. (1987) nos produtos elaborados com farinha de trigo.

Do total de fragmentos identificados, $59 \%$ corresponderam aos élitros e/ou outras áreas do exoesqueleto (cabeça, tórax e/ou abdome), 23\% foram mandíbulas, $5 \%$ corresponderam aos tarsômeros, garras e /ou esporões, $4,6 \%$ às pernas e/ou aos tarsos, $3,7 \%$ aos olhos, $3,2 \%$ às antenas, $1,3 \%$ às exúvias e $0,2 \%$ aos urogonfos larvais (Fig. 8). 

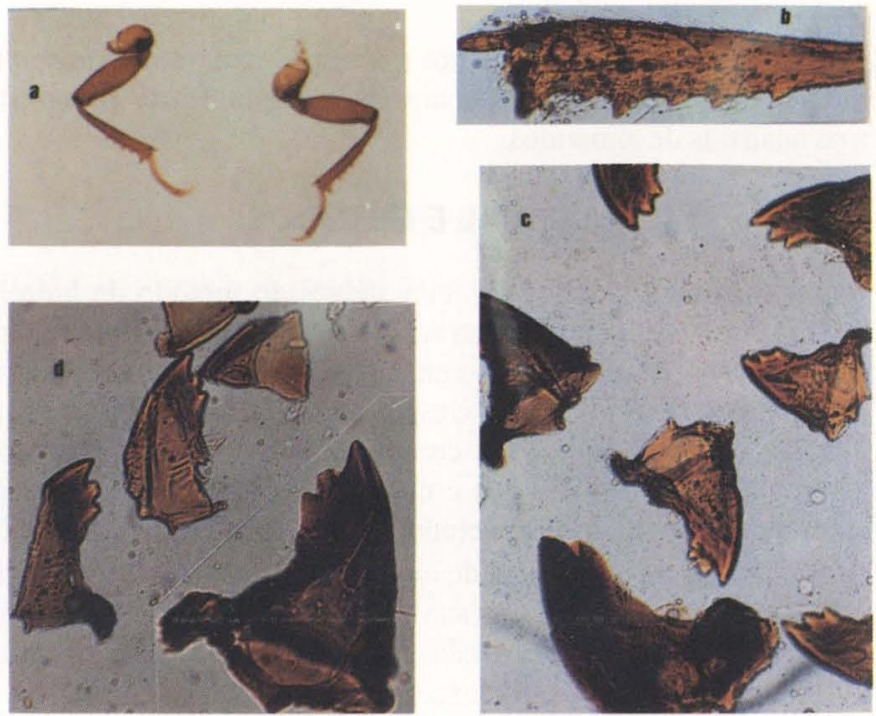

Fig. 5. Rhizopertha dominica (Fabricius, 1792) adulto. (a) Pernas completas, (b) tibia, (c) mandibulas larvais inteiras, (d) mandibula larval e fragmentos.
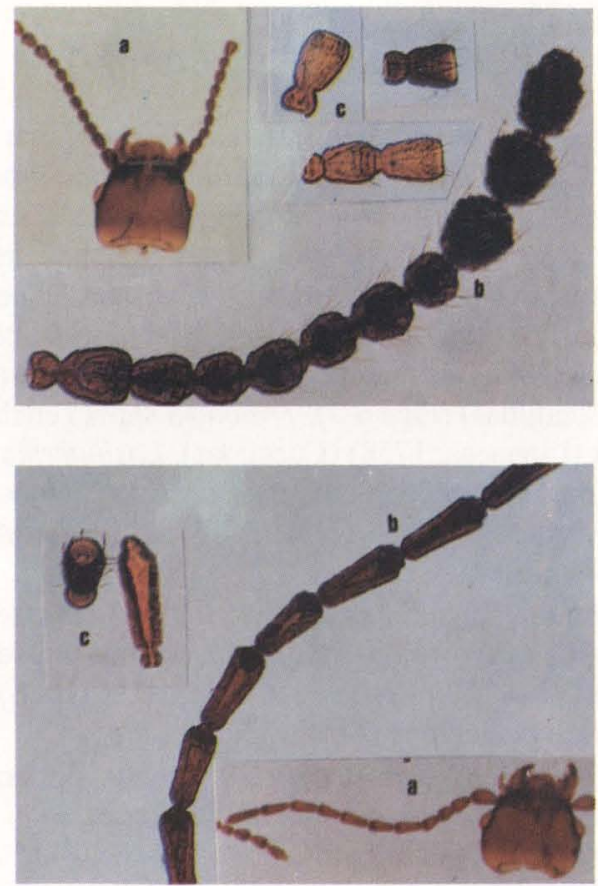

Fig. 6. Cryptolestes spp. adulto. (a) Cabeças de duas espécies, (b) antenas, (c) antenômeros. 
O tamanho médio e o intervalo de variação, dos fragmentos extraídos das amostras de farinha de trigo do tipo comum e do tipo especial, assim como daqueles extraidos das amostras de massas alimentícias (macarrão), analisadas nesta pesquisa, estão expressos na tabela I.

Tabela I. Tamanho médio dos fragmentos de insetos $(\mathrm{mm})$ e intervalo de variação dos fragmentos extraídos das farinhas de trigo comum, especial e de massas alimentícias (macarrão).

\begin{tabular}{|c|c|c|c|c|}
\hline \multirow{2}{*}{ Tipo de fragmento } & \multicolumn{2}{|c|}{ Comum } & \multicolumn{2}{|c|}{ Especial } \\
\hline & Comprimento & Intervalo variaçăo & Comprimento & Intervalo variaçăo \\
\hline $\begin{array}{l}\text { Fragmentos de élitros e de outras } \\
\text { áreas do exoesqueleto }\end{array}$ & $0,28 \pm 0,08$ & $0,04-0,56$ & $0,21 \pm 0,01$ & $0,06-0,60$ \\
\hline Antena & $0,31 \pm 0,02$ & $0,12-0,68$ & $0,36 \pm 0,02$ & $0,18-0,64$ \\
\hline Mandíbula & $0,15 \pm 0,01$ & $0,06-0,60$ & $0,14 \pm 0,01$ & $0,06-0,24$ \\
\hline Perna & $0,31 \pm 0,02$ & $0,12-0,70$ & $0,31 \pm 0,02$ & $0,14-0,60$ \\
\hline Tarso (tarsômeros) & $0,21 \pm 0,01$ & $0,04-0,44$ & $0,22 \pm 0,01$ & $0,08-0,54$ \\
\hline Fragmentos em geral * & $0,25 \pm 0,03$ & $0,04-0,70$ & $0,24 \pm 0,01$ & $0,06-0,06$ \\
\hline Fragmentos em geral & \multicolumn{4}{|c|}{$\begin{array}{l}\text { Massas alimentícias (macarrăo) } \\
0,23 \pm 0,02 \\
\text { Intervalo de variação: } 0,06-0,58\end{array}$} \\
\hline
\end{tabular}

* Fragmentos de élitros, mandibulas, antenas, pernas, tarsos e tarsômeros.

De um modo geral, os fragmentos de insetos recuperados das amostras de farinha do tipo comum, apresentaram um tamanho médio maior, do que o apresentado pelas amostras do tipo especial, exceto as mandíbulas e o tarso (tarsômeros).

Os intervalos de variação apresentados, revelaram-se de um modo geral, mais amplos nas amostras de farinha do tipo comum $(0,04$ a $0,70 \mathrm{~mm})$, quando comparados com os valores apresentados pelas amostras do tipo especial $(0,06 \mathrm{a}$ $0,64 \mathrm{~mm}$ ). Esta diferença de tamanho, entre ambos os tipos, deve ser atribuída à diferença das dimensões das malhas utilizadas no sistema de peneiração pelos moinhos. Foi observado, também, que os tamanhos médios dos fragmentos do corpo do inseto, extraídos de ambos os tipos de farinhas, apresentaram-se na maioria dos casos levemente superiores ao tamanho dos fragmentos, caracterizado pela HEALTH PROTECTION BRANCH (1984), $(<0,2 \mathrm{~mm})$, como sendo provenientes de infestação pré-moagem.

Em relação ao tamanho médio dos fragmentos recuperados das amostras de macarrão de diferentes marcas analisadas, constatou-se que este valor $(0,23 \pm 0,02 \mathrm{~mm})$ com um intervalo de variação de 0,06 a $0,58 \mathrm{~mm}$, caracteriza uma contaminação pré-moagem da matéria prima, estes valores são, também, inferiores ao tamanho médio dos fragmentos de insetos, considerado pela HEALTH PROTECTION BRANCH (1984), como sendo provenientes de infestação pré-moagem.

\section{CONCLUSÕES}

A identificação dos fragmentos de insetos, revelou que a maioria pertence as espécies do gênero Sitophilus, a Rhizopertha dominica e a Cryptolestes spp., provenientes principalmente, dos élitros e/ou outras áreas do exoesqueleto e com 



Fig. 7. Cryptolestes spp. adulto. (a) Perna completa, (b) mesosterno e metasterno, (c) fragmentos da perna.
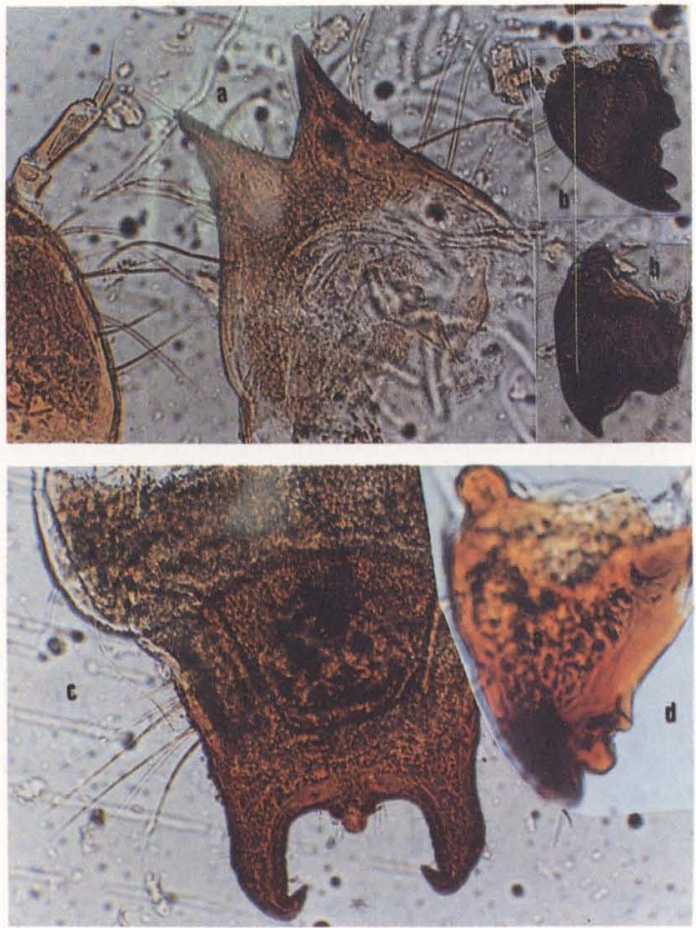

Fig. 8. Fragmentos larvais. (a) Urogonfo e (b) mandíbulas da larva de Tribolium spp.; (c) urogonfo e (d) mandíbula da larva de Cryptolestes spp. 
certa frequiência mandíbulas, em menor porcentagem tarsômeros, garras e/ou esporões, pernas e/ou tarsos, olhos, antenas, exúvias e urogonfos larvais.

O tamanho médio dos fragmentos de insetos recuperados da farinha de trigo comum, foi levemente maior do que na farinha de trigo especial $(0,25 \pm 0,03 \mathrm{~mm}$ e $0,24 \pm 0,01 \mathrm{~mm}$, respectivamente) e do macarrão foi $0,23 \pm 0,02 \mathrm{~mm}$, o que caracterizou uma infestação pré-moagem da matéria prima.

\section{REFERÊNCIAS BIBLIOGRÁFICAS}

AOAC. 1990. Official Methods of Analysis of the Association of Official Analytical Chemists. Washington, Association of Official Analytical Chemistsa, $15^{\text {th }}$ ed., p.369-406.

Bonafaccia, G.; R. CUBAdDa; V. Galli \& L.A. PASQUI. 1987. Indagine sulle impurità solide (filth test) in paste di semola di grano duro di produzione nazionale. Nota 1. Técnica Molitora 38 (3): 180-201.

GECAN, J.S. \& J.C. ATKINSON. 1985. Microanalytical quality of macaroni and noodles. J. Food Protec. 48 (5): 400-2.

Gorham, J.R. 1975. Filth in food implications for health. J. Milk Food Technol. 38 (7): 409-18.

1977. Training manual for analytical entomologuy in food industry. Washington, Food and Drug Administration, Assoc. Anal. Chem., 134p.

1979. The significance for human heath of insects in food. Ann. Rev. Entomol. 24: 209-24.

HARRIS, K.L. 1950. Identification of insect contaminants of food by the micromorphology of the insects fragments. J. Assoc. Agric. Chem. 33 (3): 898-933.

Health Protection Branch. 1984. Guidelines for Extraneous material in food. Health and Welfare Canada, 5p.

HEUERMANN, R.F. \& O.L. KURTZ. 1955. Identification of stored products insects by the micromorphology of the exoeskeleton. I. Elytral Patterns. J. Assoc. Off. Agric. Chem. 38 (3): 766-80.

JACKSON, M.M.; A.F. RATAY \& E.J. WOZNICKI. 1956. Identification of stored products insects by micromorphology of the exoeskeleton. II. Adult antennal characters. Assoc. Off. Agric. Chem. 39 (3): 766-80.

1958. Identification of stored products insects by micromorphology of the exoeskeleton. VI. Adult and larval beetle mandibles. J. Assoc. Off. Anal. Chem. 41 (2): 466-71.

KURTZ, O.L.1956. Identification of stored products insects by the micromorphology of the exoeskeleton. III. Identification of larval fragments and their significance in sanitation analysis. J. Assoc. Off. Agic. Chem. 39 (4): 990-1014.

Locatelli, D.P. \& F. Codovilli. 1986. Considerazioni da un'indagine su fragmmenti di insetti in farine di tipo e provenienza differenti. Técnica Molitora 37 (3): 180-201.

McClymont Peace, D.M. 1985. Key for identification of mandibles of stored 
food insects. Assoc. Off. Anl. Chem., 169p.

THRASHER, J.J. \& O.L. KURTZ. 1957. Identification of stored products insects by the micromorphology of the exoeskeleton. IV. Adult legs. J. Assoc. Off. Agric. Chem. 40 (3): 73-991.

WOODBURY, J.E. 1983. Reliability of analyses for indigenous insect fragments in ground paprika. J. Assoc. Off. Anal. Chem. 66 (1): 79-80.

Recebido em 30.VI.1995; aceito em 20.XI.1996. 